\title{
Intoxicação por Cestrum laevigatum (Solanaceae) em bubalinos ${ }^{1}$
}

\author{
José Diomedes Barbosa ${ }^{2 *}$, Carlos Magno C. Oliveira ${ }^{2}$, Cleyton Pinheiro ${ }^{3}$, \\ Cinthia T.A. Lopes ${ }^{3}$, Debora Marquiore ${ }^{3}$, Marilene de Farias Brito ${ }^{4}$, Elise Miyuki \\ Yamasaki $^{5}$ e Carlos Hubinger Tokarnia ${ }^{6}$
}

\begin{abstract}
Barbosa J.D., Oliveira C.M.C., Pinheiro C., Lopes C.T.A., Marquiore D., Brito M.F., Yamasaki E.M. \& Tokarnia C.H. 2010. [Poisoning by Cestrum laevigatum (Solanaceae) in buffaloes.] Intoxicação por Cestrum laevigatum (Solanaceae) em bubalinos. Pesquisa Veterinária Brasileira 30(12):1049-1052. Central de Diagnóstico Veterinário, Universidade Federal do Pará, Rua Maximino Porpino da Silva 1000, Pirapora, Castanhal, PA 68743080, Brazil. E-mail: diomedes@ufpa.br

Based on the history and clinical and pathological data, as well as on inspection of the pastures, a mortality of buffaloes in the county of Itaguaí/RJ, Brazil, was diagnosed as poisoning by Cestrum laevigatum Schlecht., a plant of the Solanaceae family. The poisoning was reproduced in two buffaloes. Dried leaves of the shrub were administered by hand, in single doses corresponding to $20 \mathrm{~g} / \mathrm{kg}$ and $40 \mathrm{~g} / \mathrm{kg}$ of the fresh leaves, to four buffaloes of the Murrah breed. The dose corresponding to $40 \mathrm{~g} / \mathrm{kg}$ of the fresh leaves caused signs of poisoning, as apathy, anorexia, absence of rumen movements, dysmetria, excitement and aggressiveness, followed by death of the two buffaloes within 65 hours after administration. From the two buffaloes that received the corresponding dose of $20 \mathrm{~g} / \mathrm{kg}$ of the fresh plant, one presented clinical signs characterized mainly by decrease of the rumen movements, but recovered 97h22min after the administration; the other buffalo did not show symptoms of poisoning. Laboratory analyses for biochemical evaluation accused hepatic alterations. In one buffalo that died, the main macroscopic finding was an orange liver with a clear nutmeg appearance; in the second buffalo, the orange liver had no nutmeg appearance. Other alterations found in these two buffaloes were slight edema of the gall bladder wall, a slightly reddish mucous membrane of the abomasum, extensive echymoses in the endocard of the left ventricle and few petechiae in the endocard of the right ventricle; the abomasum content was slightly dry, and the large intestine had little and slightly dry contents wrapped by mucus. Histopatological examination revealed severe coagulative necrosis of the liver parenchyma in the centrolobular and intermediate lobular areas, with a halo of vacuolated hepatocytes at the periphery of the necrotic areas.
\end{abstract}

INDEX TERMS: Poisonous plants, hepatotoxic plants, Cestrum laevigatum, Solanaceae, plant poisoning, buffaloes.

\footnotetext{
${ }^{1}$ Recebido em 21 de junho de 2010.

Aceito para publicação em 10 de agosto de 2010

${ }^{2}$ Central de Diagnóstico Veterinário da Faculdade de Medicina Veterinária, Universidade Federal do Pará (UFPA), Rua Maximino Porpino da Silva 1000, Pirapora, Castanhal, PA 68740-080, Brasil. *Autor para correspondência: diomedes@ufpa.br

${ }^{3}$ Curso de Pós-Graduação, Central de Diagnóstico Veterinário da Faculdade de Medicina Veterinária, UFPA, Castanhal, PA.

${ }^{4}$ Departamento de Epidemiologia e Saúde Pública, Instituto de Veterinária, Universidade Federal Rural do Rio de Janeiro (UFRRJ), Seropédica, RJ 23890-000. E-mail: marilene@ufrrj.br

${ }^{5}$ Curso de Pós-Graduação em Ciência Veterinária, Universidade Federal Rural do Rio de Janeiro (UFRRJ), Seropédica, RJ.

${ }^{6}$ Departamento de Nutrição Animal e Pastagem, Instituto de Zootecnia, Universidade Federal Rural do Rio de Janeiro (UFRRJ), Seropédica. Bolsista do CNPq. E-mail: tokarnia @ufrrj.br
}

RESUMO.- Com base no histórico e em dados clínico-patológicos, bem como a inspeção das pastagens, foi estabelecido o diagnóstico de intoxicação por Cestrum laevigatum Schlecht. em uma mortandade de búfalos no município de Itaguaí, RJ. A intoxicação foi reproduzida em dois búfalos. Amostras de folhas dessecadas de $C$. laevigatum foram administradas manualmente por via oral a quatro bubalinos da raça Murrah, em doses únicas correspondentes a $20 \mathrm{~g} / \mathrm{kg}$ e $40 \mathrm{~g} / \mathrm{kg}$ da planta fresca. A dose correspondente a $40 \mathrm{~g} / \mathrm{kg}$ provocou o aparecimento dos sinais clínicos que consistiram principalmente em apatia, anorexia, ausência dos movimentos ruminais, dismetria, excitação e agressividade, e levaram à morte os dois animais em até 65 horas após a administração da planta. Dos outros dois bufalos que receberam a 
dose correspondente a de $20 \mathrm{~g} / \mathrm{kg}$ da planta fresca, um apresentou sinais clínicos, caracterizados principalmente por diminuição dos movimentos ruminais, e recuperou-se em 97h22min após a administração da planta; o outro não apresentou sinais clínicos. Os exames laboratoriais para avaliação bioquímica indicaram lesão hepática. Em um búfalo que morreu, as principais alterações macroscópicas foram fígado de cor alaranjada, com superfície externa e de corte com nítido aspecto de noz moscada; no outro, o fígado tinha a superfície externa e de corte de cor alaranjada, sem aspecto de noz moscada. Outras alterações encontradas nos dois búfalos foram leve edema da parede da vesícula biliar, endocárdio do ventrículo esquerdo com equimoses extensas e endocárdio do ventrículo direito com algumas petéquias; mucosa do abomaso levemente avermelhada e conteúdo levemente ressecado; intestino grosso com pouco conteúdo, levemente ressecado e envolto por muco. Os exames histopatológicos revelaram no fígado, acentuada necrose de coagulação dos hepatócitos nas zonas centrais e intermediárias dos lóbulos. Na periferia dessas regiões necrosadas observou-se um halo de hepatócitos com vacuolização.

TERMOS DE INDEXAÇÃO: Bubalino, Plantas tóxicas, plantas hepatotóxicas, Cestrum laevigatum, Solanaceae, intoxicação por planta, búfalos.

\section{INTRODUÇÃO}

Plantas tóxicas constituem uma das maiores causas de prejuízos econômicos na pecuária brasileira. No Brasil, as plantas hepatotóxicas estão entre as de maior significado, no que diz respeito à grande variedade de espécies e aos prejuízos econômicos determinados pelas mortes de animais. Dentre essas, Cestrum laevigatum, um arbusto da família Solanaceae, popularmente conhecido como "coerana", "canema", "baúna", "dama-da-noite", "pimenteira", "mariapreta" e "esperto", pode ser considerada uma das mais importantes, pela sua ampla distribuição e perdas econômicas que provoca. Esta planta é encontrada nas regiões Sudeste, Centro-Oeste e na faixa litorânea da região Nordeste do Brasil. É a planta tóxica mais importante para bovinos no estado do Rio de Janeiro e, possivelmente na região sudeste. É encontrada em áreas não alagadas e em grotas. (Tokarnia et al. 2000).

A intoxicação por $C$. laevigatum, sob condições naturais tem sido registrada somente em bovinos. Dois fatores, a fome dos animais e a brotação do arbusto, são determinantes para que os animais ingiram quantidades suficientes da planta para causar intoxicação. (Tokarnia et al. 2000).

Sob condições naturais só ocorre a intoxicação aguda, que foi reproduzida em bovinos com doses únicas de 10$50 \mathrm{~g} / \mathrm{kg}$ da planta fresca. Os sinais da intoxicação são observadas 15-24h após a ingestão da planta e a morte ocorre entre 6 e 48h após o aparecimento dos sinais clínicos (Döbereiner et al. 1969).

O quadro clínico da intoxicação caracteriza-se por anorexia, apatia, tremores musculares, excitação, agressividade, andar cambaleante, constipação, fezes ressecadas con- tendo muco e sangue, gemidos, sialorreia, focinho seco, sonolência e decúbito esternal. Observam-se, também, tremores musculares, andar desequilibrado, agressividade, decúbito lateral, movimentos de pedalagem e morte. À necropsia, a lesão mais importante é encontrada no fígado, cuja superfície de corte apresenta aspecto de noz moscada (Thorburn 1934, Döbereiner et al. 1969, Lugt et al. 1991, 1992). Ao exame histológico, o fígado evidencia marcada distrofia caracterizada por necrose centrolobular associada a congestão e hemorragias, e às vezes com vacuolização dos hepatócitos na periferia da lesão (Döbereiner et al. 1969).

O objetivo desse estudo foi relatar a corrência de um surto de intoxicação por Cestrum laevigatum em búfalos e de reproduzir a intoxicação nessa espécie animal.

\section{MATERIAL E MÉTODOS}

No surto de intoxicação em búfalos no município de Itaguaí/ RJ, foi obtido o histórico da doença e foi realizada a necropsia de dois animais, com coleta de material para exames histopatológicos. A reprodução da intoxicação com as folhas de Cestrum laevigatum Schlecht. em búfalos foi realizada na Fazenda Curicaca, Castanhal/PA, no período de 12-23 de fevereiro de 2008. Foram utilizados quatro búfalos, machos, da raça Murrah, com 1-2 anos de idade. Estes eram mantidos em piquete com Brachiaria brizantha e água à vontade. Aprox. 12 horas antes da administração da planta, os animais foram separados em um curral e deixados em jejum. Antes do início do experimento, foram realizados a pesagem dos animais (o peso variou de 231 a $329 \mathrm{~kg}$ ), o exame clínico e coleta de sangue para provas de função hepática. Ao exame clínico, foram verificados os aspectos comportamentais, as frequências cardíaca e respiratória, a frequência e a intensidade dos movimentos ruminais, a temperatura retal e a cor das mucosas.

Os experimentos foram realizados com a planta dessecada. Sabe-se que as folhas de $C$. laevigatum conservam integralmente a toxidez por pelo menos um ano, quando guardadas em sacos de pano, à temperatura ambiente e à sombra (Tokarnia et al.1994). A planta usada nos experimentos foi coletada nos dias 10 e 11 de janeiro de 2008, no município de Seropédica/RJ. Tratava-se de folhas da brotação da planta que foram dessecadas (relação folha dessecada:folha verde = $1: 4)$, e posteriormente trituradas em moinho de martelo. Durante a administração aos animais eram umidificadas. Doses previamente determinadas eram colocadas na boca dos animais, que prontamente as mastigavam e deglutiam. Os Búfalos 322 e 204 receberam doses únicas da planta dessecada correspondentes a $20 \mathrm{~g} / \mathrm{kg}$ da planta fresca, e os Búfalos F1 e F2 receberam doses únicas da planta dessecada correspondentes a $40 \mathrm{~g} / \mathrm{kg}$ da planta fresca. Em seguida à administração da planta os animais eram soltos no piquete onde eram observados. Doze horas após a administração da planta foi realizado o primeiro exame clínico e coleta de sangue, e estes eram repetidos a cada quatro horas; nos intervalos dos exames clínicos os animais eram continuamente observados.

A coleta de sangue foi feita por meio de venopunção da jugular, utilizando-se frascos sem anticoagulante para a obtenção da fração sérica para as provas bioquímicas. As amostras foram enviadas para o Laboratório de Patologia Clínica da Universidade Federal do Pará, na Central de Diagnóstico Veterinário (Cedivet), para a realização dos exames. A atividade 
das enzimas Aspartato Aminotransferase (AST), ã-Glutamiltransferase (GGT), e os teores séricos de bilirrubina total e direta foram mensurados utilizando-se reagentes comerciais $\left(\right.$ Bioclin ${ }^{\circledR}$ ). As leituras dos parâmetros bioquímicos foram realizadas em analisador bioquímico semiautomático Bio Plus ${ }^{\circledR}$, modelo Bio-2000.

No caso de morte, era realizada imediatamente a necropsia e a coleta de fragmentos dos principais órgãos; estes eram fixados em solução de formol a $10 \%$ para exames histopatológicos. O material foi processado rotineiramente e corado pela Hematoxilina-Eosina (HE), no Setor de Anatomia Patológica (SAP) do Projeto Sanidade Animal Embrapa/UFRRJ.

\section{RESULTADOS}

\section{Surto de intoxicação natural}

O rebanho de búfalos onde ocorreu a mortandade, no município de Itaguaí/RJ, era constituído de aproximadamente 150 animais, entre adultos e jovens. Os animais eram pastoreados nas margens das estradas e em loteamentos em fase de instalação. Por ocasião da nossa consulta, em 18 de agosto de 1991, já haviam morrido alguns animais em dias anteriores e havia dois animais recém-mortos.

Os dados dos dois búfalos recém-mortos necropsiados constam abaixo:

Necropsia 11/91. Búfala, fêmea, com aprox. 3 anos de idade. Histórico: na noite anterior a búfala mostrou tremores e berrava muito. Amanheceu caída, viva. Morreu aprox. às 8 horas. Achados de necropsia (necropsia realizada às 10:00h): Superfície de corte do fígado com acentuado aspecto de noz moscada. Conteúdo do omaso acentuadamente ressecado. Conteúdo do cólon e do reto levemente ressecado e em forma de esferas com muco e sangue. Os exames histopatológicos (SAP 25458) revelaram, no fígado, acentuada necrose de coagulação nas zonas centrais e intermediárias dos lóbulos, associada a congestão e vacuolização dos hepatócitos na periferia das áreas de necrose. Nos hepatócitos ao redor dos espaços porta havia presença de vacúolos pequenos, em grau discreto a leve.

Necropsia 12/91. Búfalo, macho, com aproximadamente 2 anos de idade. Histórico: pela manhã o animal saiu bem para pastar; às 11:30h foi encontrado caído e à 13:30 estava morto. Achados de necropsia: Fígado com acentuada congestão; a superfície de corte apresentava aspecto de noz moscada. A parede da vesícula biliar apresentava edema moderado. No endocárdio havia equimoses. O conteú- do do omaso estava acentuadamente ressecado, e o do cólon e do reto estava levemente ressecado e em forma de esferas com muco e sangue. Os exames histopatológicos (SAP 25459) revelaram, no fígado, acentuada necrose de coagulação nas zonas centrais e intermediárias dos lóbulos associada à congestão. Nos hepatócitos, ao redor do espaço porta, havia pequenos vacúolos, em grau discreto a leve.

À inspeção das áreas onde o rebanho de búfalos pastava, verificou-se grande quantidade de arbustos de Cestrum laevigatum, com sinais de terem sido pastados. Havia escassez de pastagem.

\section{Experimentação}

No Quadro 1 consta o delineamento dos experimentos, com os principais dados.

As folhas dessecadas de $C$. laevigatum, na dose correspondente a $20 \mathrm{~g} / \mathrm{kg}$ das folhas frescas causou sinais clínicos discretos em um animal (Búfalo 322), enquanto que a dose correspondente a $40 \mathrm{~g} / \mathrm{kg}$ da planta fresca causou a morte dos dois animais (Búfalos F2 e F1).

Nos búfalos que morreram, os primeiros sinais de intoxicação foram observados entre 23h40min (Búfalo F1) e 26h05min (Búfalo F2) após o início da administração da planta e a evolução foi de 24h11 min a 41h19min.

No Búfalo 322 que adoeceu e se recuperou, observouse apenas diminuição dos movimentos ruminais. Os sinais clínicos nos dois búfalos que morreram foram: apatia, anorexia, diminuição ou ausência dos movimentos ruminais, sialorreia, dificuldade na inspiração, andar cambaleante, dismetria, excitação, agressividade, constipação, com fezes ressecadas contendo muco e sangue, gemidos, focinho seco, sonolência, decúbito lateral, movimentos de pedalagem e morte.

No Búfalo $F 1$, as principais alterações macroscópicas foram fígado de cor alaranjada, com superfície externa e de corte com nítido aspecto de noz moscada. No Búfalo F2, o fígado tinha a superfície externa e de corte de cor alaranjada, sem aspecto de noz moscada. Outras alterações nos dois búfalos eram leve edema da parede da vesícula biliar, mucosa do abomaso levemente avermeIhada, endocárdio do ventrículo esquerdo com equimoses extensas e endocárdio do ventrículo direito com algumas petéquias, conteúdo do abomaso levemente ressecado, e o intestino grosso com pouco conteúdo, levemente ressecado e envolto por muco. Os exames histopatológicos re-

Quadro 1. Delineamento dos experimentos em búfalos com as folhas dessecadas de Cestrum laevigatum

\begin{tabular}{|c|c|c|c|c|c|c|}
\hline $\begin{array}{l}\text { Identificação } \\
\text { dos búfalos }\end{array}$ & $\begin{array}{l}\text { Peso } \\
(\mathrm{kg})\end{array}$ & $\begin{array}{l}\text { Dose única } \\
\text { correspondente } \\
\text { à planta fresca }\end{array}$ & $\begin{array}{c}\text { Data e hora } \\
\text { da administração }\end{array}$ & $\begin{array}{l}\text { Início dos sinais } \\
\text { clínicos após } \\
\text { a administração }\end{array}$ & Evolução & $\begin{array}{c}\text { Recuperação ou } \\
\text { morte após a } \\
\text { administração } \\
\text { da planta }\end{array}$ \\
\hline 322 & 295 & $20 \mathrm{~g} / \mathrm{kg}$ & $\begin{array}{c}12.2 .08 \\
\text { 7h30-9h50 e } 17 \mathrm{~h}-18 \mathrm{~h} 08\end{array}$ & $37 \mathrm{~h} 22 \mathrm{~min}$ & $60 \mathrm{~h}$ & $\begin{array}{l}\text { 97h22min } \\
\text { Recuperou-se }\end{array}$ \\
\hline 204 & 309 & $20 \mathrm{~g} / \mathrm{kg}$ & $\begin{array}{c}\text { 19.2.08 } \\
\text { 09h30-13h15 }\end{array}$ & Sem sinais & - & - \\
\hline F2 & 231 & $40 \mathrm{~g} / \mathrm{kg}$ & $\begin{array}{c}12.2 .08 \\
10 h 30-16 h 35\end{array}$ & $26 \mathrm{~h} 05 \mathrm{~min}$ & $\begin{array}{l}\text { 24h11min } \\
\text { Morreu (SAP 31207) }\end{array}$ & 50h16min \\
\hline $\mathrm{F} 1$ & 329 & $40 \mathrm{~g} / \mathrm{kg}$ & $\begin{array}{c}\text { 19.2.08, } \\
\text { 09h30-13h50 }\end{array}$ & 23h40min & $\begin{array}{l}\text { 41h19min } \\
\text { Morreu (SAP 31205/06) }\end{array}$ & 64h59min \\
\hline
\end{tabular}


velaram no fígado acentuada necrose de coagulação dos hepatócitos nas zonas centrais e intermediárias dos lóbulos. Na periferia dessas regiões necrosadas observou-se um halo de hepatócitos com vacuolização.

Nas análises clínicas, os dois animais (Búfalos 322 e 204) que receberam a planta dessecada na dose correspondente a $20 \mathrm{~g} / \mathrm{kg}$ da planta fresca, tinham a média das enzimas $\gamma$-Glutamil-transferase (GGT), Aspartato aminotransferase (AST); os valores da bilirrubina total antes da administração da planta foram 16,33 U/L, 148,5 U/L e $0,37 \mathrm{mg} / \mathrm{dl}$, respectivamente, e passaram para $128,3 \mathrm{U} / \mathrm{L}$, 379,16 e 0,84 após a ingestão da planta.

Nos dois animais (Búfalos 322 e 201) que receberam a planta dessecada na dose correspondente a $20 \mathrm{~g} / \mathrm{kg}$ da planta fresca, a média das enzimas $\gamma$-Glutamil-transferase (GGT), Aspartato-aminotransferase (AST) e bilirrubina total antes da administração da planta foram 16,33 U/L, 148,5 $\mathrm{U} / \mathrm{L}$ e $0,37 \mathrm{mg} / \mathrm{dl}$, respectivamente, e passaram para 128,3 $\mathrm{U} / \mathrm{L}, 379,16 \mathrm{U} / \mathrm{L}$ e 0,84mg/dl após a ingestão da planta.

\section{DISCUSSÃO}

Em experimentos realizados no Brasil (Döbereiner et al. 1969), doses de $10-24 \mathrm{~g} / \mathrm{kg}$ causaram a morte de $50 \%$ dos bovinos, enquanto as doses de $25-50 \mathrm{~g} / \mathrm{kg}$ causou a morte de $80 \%$ dos bovinos. A dose tóxica variou muito de um animal para outro, sendo que alguns bovinos morreram com doses de $10 \mathrm{~g} / \mathrm{kg}$, enquanto que outros resistiram a doses de até $50 \mathrm{~g} / \mathrm{kg}$; doses abaixo de $10 \mathrm{~g} / \mathrm{kg}$ não foram tóxicas para os bovinos. Em experimentos realizados na África do Sul (Lugt et al. 1991, 1992), doses de 8 e $10 \mathrm{~g} / \mathrm{kg}$ foram letais para 2 de 3 bovinos e 2 de 4 ovinos, o que indica a eventual maior toxidez da planta na África do Sul em relação à encontrada no Brasil.

As provas de função hepática revelaram um aumento nas concentrações séricas das enzimas $\gamma$-Glutamiltransferase (GGT) e Aspartato-aminotransferase (AST) em todos os animais do experimento, após a ingestão da planta, independente da dose recebida. A elevação da GGT, que é uma enzima originária das membranas dos canalículos e ductos biliares, caracterizou o quadro de colestase, uma vez que o aumento dessa enzima se relaciona exclusivamente à colestase intra e extra-hepática e com proliferação de ductos biliares (Santos et al. 2008). A elevação da concentração sérica da enzima Aspartato-aminotransferase (AST), corroborou para o diagnóstico de lesão hepatocítica, pois o aumento da atividade sérica dessa enzima pode ser causado por necrose e também por lesão subletal de hepatócitos (Cardoso 2008).

O aumento da concentração sérica de bilirrubina, obser- vado nos quatro búfalos, correlaciona-se à lesão hepática e do trato biliar, o que influencia na queda da excreção desse metabólito (Santos et al. 2008). A obstrução do fluxo biliar ocasionada pela colestase resulta na passagem de bilirrubina conjugada para o sangue, o que é denominado de hiperbilirrubinemia de regurgitação, hiperbilirrubinemia pós-hepática ou colestática (Thrall et al. 2007).

\section{CONCLUSÕES}

Bubalinos são sensíveis à intoxicação por Cestrum laevigatum.

O quadro clínico-patológico da intoxicação por $C$. laevigatum em bubalinos é semelhante ao observado em bovinos.

As provas de função hepática mostraram-se eficientes na detecção das lesões hepáticas de búfalos intoxicados por C. laevigatum.

Dose da planta dessecada correspondente a $20 \mathrm{~g} / \mathrm{kg}$ da planta fresca causou sinais clínicos discretos ou ausentes.

Dose da planta dessecada correspondente a $40 \mathrm{~g} / \mathrm{kg}$ da planta fresca foi letal para os bubalinos.

\section{REFERÊNCIAS}

Cardoso C.A. 2008. Comparação de kits comerciais na dosagem de constituintes bioquímicos do sangue em equinos hígidos. Dissertação de Pós-Graduação em Medicina Veterinária. Universidade Federal de Viçosa, Viçosa, MG. 143p.

Döbereiner J., Tokarnia C.H. \& Canella C.F.C. 1969. Intoxicação por Cestrum laevigatum Schlecht., a causa de mortandades em bovinos no Estado do Rio de Janeiro. Pesq. Agropec. Bras. 4:165-93.

Lugt J.J., Van der Nel P.W. \& Kitcfflng J.P. 1991. The pathology of Cestrum laevigatum (Schlecht.) poisoning in cattle. Onderstepoort J. Vet. Res. 58:211-221.

Lugt J.J., Van der Nel P.W. \& Kitcfflng J.P. 1992. Experimentally-induced Cestrum laevigatum (Schlecht.) poisoning in sheep. Onderstepoort J. Vet. Res. 59:135-144.

Santos J.C.A., Riet-Correa F., Simões S.V.D. \& Barros C.S.L. 2008. Patogênese, sinais clínicos e patologia das doenças causadas por plantas hepatotóxicas em ruminantes e equinos no Brasil. Pesq. Vet. Bras. 28(1):1-14.

Thorburn J.A. 1934. Chase Valley Disease: Cestrum laevigatum Schlecht., its toxic effects on ruminants. Onderstepoort J. Vet. Sci. Anim. Indust. 2(2):667-679.

Thrall M.A., Baker C.D., Campbell T.W., DeNicola D., Fettman M.J., Lassen E.D., Rebar A. \& Weiser G. 2007. Hematologia e Bioquímica Clínica Veterinária. Roca, São Paulo. 582p.

Tokarnia C.H., Döbereiner J. \& Peixoto P.V. 1994. Aspectos clínicopatológicos complementares da intoxicação por algumas plantas tóxicas brasileiras. Pesq. Vet. Bras. 14(4):111-122.

Tokarnia C.H., Döbereiner J. \& Peixoto P.V. 2000. Plantas Tóxicas do Brasil. Editora Helianthus, Rio de Janeiro, p.80-109. 